\title{
Generating Difference Equations with the Darboux Transformation
}

\author{
Michael Reach \\ 1541 E $5^{\text {th }}$ Street, Brooklyn, NY 11230, USA
}

\begin{abstract}
Starting with a system satisfying the so-called "bispectral property," a method is given to generate other systems with still higher-order recursion relations. The usefulness of the method is checked for the classical examples.
\end{abstract}

\section{Introduction}

For many well-known functions arising as eigenfunctions of ordinary differential equations, an important and common additional property is the existence of a recursion relation in the spectral parameter. This will always happen for orthogonal polynomials, but in practice it is also found for virtually anything with a name, as examination of books on the subject of classical functions will attest. For the purpose of practical computation, this property is beyond price.

In [5], Duistermaat and Grünbaum classified all sets of eigenfunctions satisfying differential operators in two different independent variables, the bispectral property. The partially discrete version of this, to classify all differential operators whose eigenfunctions also satisfy recursion relations, is a problem that remains open (see [7]). In this paper, the question is approached from a slightly different angle: Starting with a differential operator whose eigenfunctions also satisfy a recursion relation, a constructive method is given to generate other differential operators with the same property. This give hope of solving the classification problem in a different way: by showing that all solutions are generated from a small number of primitives.

1.1. Notation. We start with a quintuple $(L, B, \lambda, \Theta, \phi)$ satisfying

and

$$
L \phi_{n}(x)=\lambda_{n} \phi_{n}(x)
$$

$$
(B \phi)_{n} \equiv \sum_{i=-N}^{N} b_{i}^{(n)} \phi_{n+i}(x)=\Theta(x) \phi_{n}(x) .
$$

Here $\partial \equiv d / d x \cdot L \equiv \partial^{2}+V(x)$ is a formal differential operator in one variable $x$, and $B$ is a difference operator in $n$. The formula (2) is an example of a recursion relation, since it provides a way to generate $\phi_{n+N}$ given the $\phi$ 's for smaller $n$. In this paper, the eigenvalue $\Theta$ is assumed to be independent of $n$, and the $b_{i}$ 's independent of $x . n$ will range over the integers. 
What is sought are transformations of this quintuple into new ones that also will satisfy these two equations.

The particular form sought here for the difference operator $B$ is based on the usual one for the three-term relations always satisfied by orthogonal polynomials:

$$
x p_{n}=a_{1}^{(n)} p_{n+1}+a_{0}^{(n)} p_{n}+a_{-1}^{(n)} p_{n-1} .
$$

Obviously, many other kinds of recursion relations can exist. The only true practical requirement is that the coefficients be, in some sense or another, "easier" to calculate than the eigenfunctions themselves. This rescues the problem from being vacuous. Seeking functions satisfying more general recursion relations (along with a differential equation in $x$ ) might be a good generalization of the work here.

Duistermaat and Grünbaum discuss a similar question in [5], where they replace the difference operator $B$ by a differential operator in $\lambda$. Thus, the notation used throughout will also be similar, and many of the introductory results are lifted bodily from there.

The reader will notice that many usual features of functional analysis are missing: boundary conditions, complete sets of eigenfunctions, and so forth. The reason is that, in its essence, the problem is purely local, and should be so handled: All that matters is that our desired properties hold near some $x_{0}$ and for "a lot of integers" near some $n_{0}$. Therefore, we never ought to have to give boundary conditions, but only smoothness requirements on the functions and coefficients involved.

The notation for a simple transformation will be introduced here, since it is to be used throughout the paper. We will usually call it "unravelling." Say that a set of eigenfunctions $\phi_{n}$ satisfies (1). Then an operator $\mathcal{O}\left(x, \partial, \lambda_{n}\right)$ depending on $n$ can often be changed to a new operator $\mathcal{O}(x, \partial, L)$ with the $n$-dependence removed, but giving exactly the same result when operating on any of the $\phi_{n}$ 's. This works properly when $\mathcal{O}$ is a sum of terms of the form $p_{i}\left(\lambda_{n}\right) \mathbf{O}_{i}(x, \partial)$ with the $p_{i}$ 's polynomials. Then

$$
p_{i}\left(\lambda_{n}\right) \mathbf{O}_{i}(x, \partial) \phi_{n}(x)=\mathbf{O}_{i}(x, \partial) p_{i}\left(\lambda_{n}\right) \phi_{n}(x)=\mathbf{O}_{i}(x, \partial) p_{i}(L) \phi_{n}(x)
$$

gives the unravelling of one of the terms. Note that the parts dependent on $n$ must always be moved to the right. This transformation will be called $\mathscr{U}$, so that

$$
\mathscr{U}\left(\sum_{i} p_{i}\left(\lambda_{n}\right) \mathbf{O}_{i}(x, \partial)\right)=\sum_{i} \mathbf{O}_{i}(x, \partial) p_{i}(L) .
$$

If dependence on $\lambda_{n}$ is more complicated, unravelling may be impossible to do completely, unless pseudo-differential operators are used.

1.2. Morphisms. There are many obvious transformations of a solution to our problem that leave it a solution. The following three will be very useful to us in what follows.

1. The first morphism is simply a change in the independent variable $x$. Let $x=x\left(x^{\prime}\right)$. If $L^{\prime}$ is the new differential operator resulting from the variable change, we get the new solution

$$
\left(L^{\prime}, B, \lambda, \Theta\left(x\left(x^{\prime}\right)\right), \phi\left(x\left(x^{\prime}\right)\right)\right)
$$


(1) and (2) change in the obvious ways. One example of this often used is the simple shift $x=x^{\prime}+c^{\prime}$.

2. Let $\Psi_{n}(x)=f(x) \phi_{n}(x)$ for some function $f(x)$ and for all $n$. Then

$$
\left(f L f^{-1}, B, \lambda, \Theta, \psi, c\right)
$$

is a solution, allowing us to multiply $\phi$ by an arbitrary function of $x$. (2) looks similar, but with $\psi$ in place of $\phi$, and (1) becomes $\left(f L f^{-1}\right) \psi_{n}(x)=\lambda_{n} \psi_{n}(x)$.

3. Let $\psi_{n}(x)=f_{n} \phi_{n}(x)$, for all $k$ and for $f_{n}$ independent of $x$. This multiplication of $\phi_{n}$ by an arbitrary constant depending only on $n$ gives the new solution

$$
\left(L, f B f^{-1}, \lambda, \Theta, \psi, c\right) \text {. }
$$

Here (1) will be similar, while (2) becomes $f_{n}\left(B\left(f^{-1} \psi\right)\right)_{n}=\Theta(x) \psi_{n}(x)$.

Both the morphisms mentioned leave the size of the recursion relation unchanged, and are therefore found in [6]. We cannot make a change of variables in the eigenvalue parameter $n$ in the same way as we do with $x$, because the spacing between the values of $n$ in the terms of recursion relation is fixed to be unity by definition. This is one of the reasons that the discrete problem is more difficult than its continuous counterpart.

However, an integer change in scale of the eigenvalue parameter can be made to work. In fact, this amounts to a way to generate bigger recursion relations, easier (though less interesting) than the Darboux transformation method given later in this paper.

Say $m \in \mathbf{Z}$, and that the recursion relation exists for $n \in \mathbf{Z} / m+c$. Setting the parameter $n^{\prime}$ to be $n / m$ changes the recursion relation from one of $2 N+1$ st order to one of $2 m N+1^{\text {st }}$ order. It is the same relation, of course, but now is sparse; only every $m$ th eigenfunction appears in the relation.

Note that this last morphism requires that the original recursion relation exists, not only for the eigenvalue parameter $n \in \mathbf{Z}+c$, but for all $m^{\text {th }} \mathbf{s}$ of integers: for $n \in \mathbf{Z} / m+c$. In practice, this is no restriction, and the reason is very significant. For all the examples we will find, and all the methods given to extend these to other examples, if a solution works for a sextuple with a given $c$, it will work for all $c \in \mathbf{C}$. Therefore, we can shift from the original $c$ by $1 / m, 2 / m, \ldots,(m-1) / m$ and get other recursion relations, so that this transformation goes through.

In this transformation, we need to know how the other elements of the sextuple change. The two cases important in practice will be $\lambda_{n}=n$ and $\lambda_{n}=n^{2}$. If we originally had

$$
\left(\partial^{2}+V(x)\right) \phi_{n}(x)=n \phi_{n}(x)
$$

as (1), we will now use

$$
\left(\partial^{2}+m V(\sqrt{m} x)\right) \phi_{n / m}(\sqrt{m} x)=n \phi_{n / m}(\sqrt{m} x) .
$$

Thus, the new $n$-eigenfunction $\psi_{n}(x) \equiv \phi_{n / m}(\sqrt{m} x)$.

On the other hand, if

$$
\left(\partial^{2}+V(x)\right) \phi_{n}(x)=n^{2} \phi_{n}(x)
$$


is the original equation, the changed result will be

$$
\left(\partial^{2}+m^{2} V(m x)\right) \phi_{n / m}(m x)=n^{2} \phi_{n / m}(m x),
$$

which uses the new $\psi_{n}(x) \equiv \phi_{n / m}(m x)$.

Finally, it is worth noting that this process can be reversed, if the original recursion relation happens to be properly sparse. This will actually occur in one practical example: the Bessel functions.

1.3. The Darboux Transformation and Wronskians. One of the most important tools in this line of business was introduced in 1882 by Darboux [4], Livre IV, Chap. IV, Chap. IX, No. 408. On page 52, he actually attributes it to Moutard, which may be the earliest known reference to the idea. To introduce the transformation here we will assume that $L=\partial^{2}+V(x)$ is a second-order differential operator, but the concept generalizes easily to higher orders.

If $\phi$ is an eigenfunction of $L$, eigenvalue $\lambda$, i.e.,

$$
L \phi=\lambda \phi,
$$

then we can factor $L-\lambda$ in the following way:

$$
L-\lambda=P Q \quad \text { with } \quad P=\partial+\frac{\phi^{\prime}(x)}{\phi(x)}, \quad Q=\partial-\frac{\phi^{\prime}(x)}{\phi(x)} .
$$

If one interchange $P$ and $Q$, a new operator is obtained:

with

$$
\tilde{L}=Q P+\lambda=\partial^{2}+\tilde{V}(x)
$$

$$
\tilde{V}(x)=V(x)+2\left(\frac{\phi^{\prime}(x)}{\phi(x)}\right)^{\prime}
$$

giving a new potential. This operation is called the Darboux transformation. The eigenfunctions of the new operator are given by magic: If $L \phi_{\mu}=\mu \phi_{\mu}$, then $\tilde{L} Q \phi_{\mu}=$ $(Q P+\lambda) Q \phi_{\mu}=Q L \phi_{\mu}=\mu Q \phi_{\mu}$, so that

$$
\tilde{L} \psi_{\mu}=\mu \psi_{\mu}, \quad \text { with } \quad \psi_{\mu}=\left(\partial-\frac{\phi^{\prime}}{\phi}\right) \phi_{\mu} .
$$

These new eigenfunctions are obtained directly from the old ones, just by applying Q. Burchnall and Chaundy [3] may have been the first to explain the Darboux transformation as an exchange of factors of the differential operator, and therefore called it transference. It is also often known as the Backlund transformation.

One property that we will need later is a well-known Wronskian form for eigenfunctions obtained through Darboux. To show it, we will first give two old facts about Wronskians, stated, for example, in Adler and Moser [2]. The second is an identity by Jacobi.

Let $W\left(f_{1}, f_{2}, \ldots, f_{m}\right)$ be the usual Wronskian. It can have any number of arguments.

Lemma 1. For any $f, g, h$ functions of $x$

$$
W\left(\frac{f}{h}, \frac{g}{h}\right)=\frac{1}{h^{2}} W(f, g) .
$$


Proof. Trivial.

Lemma 2 (Jacobi). If $f_{1}, f_{2}, \ldots, f_{m}, g, h$ are functions of $x$, then

$$
W\left(W\left(f_{1}, f_{2}, \ldots, f_{m}, g\right), W\left(f_{1}, f_{2}, \ldots, f_{m}, h\right)\right)=W\left(f_{1}, f_{2}, \ldots, f_{m}\right) \cdot W\left(f_{1}, \ldots, f_{m}, g, h\right) .
$$

Proof. The easiest way to prove this, as with most Wronskian identities, is by considering both sides of the equation to be differential operators acting on the function $h$. One can see immediately that the two sides have the same null space, i.e., $h=f_{1}, f_{2}, \ldots, f_{m}$, or $g$. It is easy to see that they are of the same order, and all that is left is to check that their highest-order coefficients are the same.

Say that Darboux has been done $m$ times, starting from some original $L_{0}$, and calling the resulting differential operators $L_{1}, L_{2}, \ldots, L_{m}$. We see from (3) that an eigenfunction of $L_{m}$ with eigenvalue $\lambda$ will be of the form

$$
\left(\partial+r_{1}\right)\left(\partial+r_{2}\right) \cdots\left(\partial+r_{m}\right) \phi_{\lambda}
$$

where $\phi_{\lambda}$ is a generalized $\lambda$-eigenfunction for $L_{0}$. (We may need generalized eigenfunctions, that is, functions in the null space of $(L-\lambda)^{k}$, if Darboux has been done $k-1$ times before with the same eigenvalue $\lambda$. In the more normal case where all the eigenvalues used are different, we won't have to worry about them at all.) Call this new eigenfunction "derived from $\phi_{\lambda}$." Now, the $j^{\text {th }}$ time that Darboux is repeated, it must be done with respect to some eigenfunction of $L_{j-1}$, say with eigenvalue $\alpha_{j}$. This eigenfunction is also derived from some $\phi_{\alpha_{j}}$, an eigenfunction of $L_{0}$. We see that a sequence of Darboux transformations can be characterized by the sequence of $\phi_{\alpha_{j}}$ 's eigenfunctions of the original $L_{0}$. The rather pretty fact is that

Lemma 3. Let an operator $L$ have eigenfunctions $\phi_{i}$. If a sequence of $m$ Darboux transformations are carried out using eigenfunctions derived from $\phi_{\alpha_{1}}, \phi_{\alpha_{2}}, \ldots, \phi_{\alpha_{m}}$, then an $\lambda$-eigenfunction of the resulting operator will be of the form

$$
\frac{W\left(\phi_{\alpha_{1}}, \ldots, \phi_{\alpha_{m}}, \phi_{\lambda}\right)}{W\left(\phi_{\alpha_{1}}, \ldots, \phi_{\alpha_{m}}\right)} \text {. }
$$

The proof will proceed by induction. The beginning step is clear if $W$ (no arguments) $\equiv 1$. Now, say the lemma is known to be true for the first $j-1$ Darboux steps. Then the eigenfunctions $\psi_{\lambda}$ of the new operator will be of the form (6). To do Darboux another time, let us pick one of them, say $\psi_{\alpha_{\jmath}}$, coming from $\phi_{\alpha_{j}}$. Afterwards, the new $n^{\text {th }}$ eigenfunction looks like $\left(\partial-\psi_{\alpha_{j}}^{\prime} / \psi_{\alpha_{j}}\right) \psi_{\lambda}$, which equals $W\left(\psi_{\alpha_{j}}, \psi_{\lambda}\right) / \psi_{\alpha_{j}}$. By assumption, this equals

$$
\frac{W\left(\frac{W\left(\phi_{\alpha_{1}}, \ldots, \phi_{\alpha_{j-1}}, \phi_{\alpha_{j}}\right)}{W\left(\phi_{\alpha_{1}}, \ldots, \phi_{\alpha_{l-1}}\right)}, \frac{W\left(\phi_{\alpha_{1}}, \ldots, \phi_{\alpha_{j-1}}, \phi_{\lambda}\right)}{W\left(\phi_{\alpha_{1}}, \ldots, \phi_{\gamma_{1}-1}\right)}\right)}{\frac{W\left(\phi_{\alpha_{1}}, \ldots, \phi_{\alpha_{j}-1}, \phi_{\alpha_{\jmath}}\right)}{W\left(\phi_{\alpha_{1}}, \ldots, \phi_{\alpha_{j}-1}\right)},}
$$

which by (4) is

$$
\frac{W\left(W\left(\phi_{\alpha_{1}}, \ldots, \phi_{\alpha_{j-1}}, \phi_{\alpha_{.}}\right), W\left(\phi_{\alpha_{1}, \ldots, \phi_{\alpha_{j}-1}}, \phi_{\lambda}\right)\right)}{W\left(\phi_{\alpha_{1}}, \ldots, \phi_{\alpha_{j}-1}\right) W\left(\phi_{\alpha_{1}}, \ldots, \phi_{\alpha_{j-1}}, \phi_{\alpha_{j}}\right)}
$$


By (5), this comes to be precisely the form required for the $j^{\text {th }}$ induction step.

\section{Generating Solutions with Darboux}

Already, in the section on "Morphisms," we have seen a way of generating layer recursion relations out of smaller ones, to wit, by shrinking the eigenvalue. This method is, of course, not useful for producing any solutions that look really different. Such a procedure, is however, actually available, and can be used to generate arbitrary numbers of new and interesting solutions, starting only with the classical functions that have the three-term relations.

2.1 The Wronskian Relation. A simple lemma is necessary to start this process. Throughout this section, let $f_{1}, f_{2}, \ldots, f_{m}$ and $p$ be arbitrary functions of $x$.

Lemma 4. Define

$$
\left[\begin{array}{llll}
f_{1} & f_{2} & \cdots & f_{m} \\
r_{1} & r_{2} & \cdots & r_{m}
\end{array}\right]
$$

for the $r_{i}$ 's nonnegative integers, to be the determinant of the $m \times m$ matrix whose $(a, b)$ entry is $f_{a}^{\left(r_{b}\right)}$. Then

$$
\sum_{j=1}^{m}(-1)^{j} f_{j}^{(i)}\left[\begin{array}{cccccc}
f_{1} & f_{2} & \cdots & \hat{f}_{j} & \cdots & f_{m} \\
r_{1} & r_{2} & & \cdots & & r_{m-1}
\end{array}\right]=0 \text { for } i=\text { any } r_{k} .
$$

The hat over $f_{j}$ in the equation means that the Wronskian includes all the $f$ 's but that one.

Proof. The left-hand side of the formula is simply the expansion by minors of the following determinant:

$$
\left|\begin{array}{llll}
f_{1}^{\left(r_{1}\right)} & f_{2}^{\left(r_{1}\right)} & \cdots & f_{m}^{\left(r_{1}\right)} \\
f_{1}^{\left(r_{2}\right)} & f_{2}^{\left(r_{2}\right)} & \cdots & f_{m}^{\left(r_{2}\right)} \\
\vdots & & \vdots & \\
f_{1}^{\left(r_{m-1}\right)} & f_{2}^{\left(r_{m-1}\right)} & \cdots & f_{m}^{\left(r_{m-1}\right)} \\
f_{1}^{(i)} & f_{2}^{(i)} & \cdots & f_{m}^{(i)}
\end{array}\right|
$$

An equally trivial corollary of this is the following.

\section{Corollary 1.}

$$
\sum_{j=1}^{m}(-1)^{j} f_{j}^{(i)} W\left(f_{1}, \ldots, \hat{f}_{j}, \ldots, f_{m}\right)=\left\{\begin{array}{cl}
0, & i=0,1, \ldots, m-2, \\
(-1)^{m} W\left(f_{1}, f_{2}, \ldots, f_{m}\right), & i=m-1
\end{array}\right.
$$

Using this formula we can prove the following interesting proposition.

Proposition 1. Let $p(x)$ be another arbitrary function.

$$
\begin{aligned}
& W\left(f_{1}, f_{2}, \ldots, f_{N+1}\right) \int p W\left(f_{1}, f_{2}, \ldots, f_{N}\right) \\
& \quad=W\left(f_{1}, \ldots, f_{N}, \sum_{j=1}^{N+1}(-1)^{N+1+j} f_{j} \int p W\left(f_{1}, \ldots, \hat{f}_{j}, \ldots, f_{N+1}\right)\right) .
\end{aligned}
$$


Though there are several ways to prove this fact, the following will help to clarify its origin. The right-hand side, written as a full determinant, is

$$
\left|\begin{array}{cccc}
f_{1} & \cdots & f_{N} & \sum(-1)^{N+1+j} f_{j} \int p W\left(f_{1}, \ldots, \widehat{f}_{j}, \ldots, f_{N+1}\right) \\
f_{1}^{\prime} & \cdots & f_{N}^{\prime} & ()^{\prime} \\
\vdots & \vdots & & \vdots \\
f_{1}^{(N)} & \cdots & f_{N}^{(N)} & ()^{(N)}
\end{array}\right|
$$

Now, the $(2, m+1)$ entry of this array, which we wrote as ()$^{\prime}$, will have two terms,

$$
\begin{aligned}
& \sum_{j}(-1)^{N+1+j} f_{j}^{\prime} \int p W\left(f_{1}, \ldots, \hat{f}_{j}, \ldots, f_{N+1}\right) \\
& \quad+p \sum_{j}(-1)^{N+1+j} f_{j} W\left(f_{1}, \ldots, \hat{f}_{j}, \ldots, f_{N+1}\right) .
\end{aligned}
$$

However, the second term vanishes by (8). Thus, the $(2, m+1)$ entry really only is the one term $\sum(-1)^{N+1+j} f_{j}^{\prime} \int p W\left(f_{1}, \ldots, \hat{f}_{j}, \ldots, f_{N+1}\right)$. Continuing to the $(3, m+1)$ entry, we see now that it also will consist of only two terms, and again one will vanish. Going on thus, the $(i, m+1)$ entry for $i=1, \ldots, m+1$ simply turns out to be

$$
W_{i, m+1}=\sum(-1)^{N+1+j} f_{j}^{(i-1)} \int p W\left(f_{1}, \ldots, \hat{f}_{j}, \ldots, f_{N+1}\right) .
$$

The right-hand-side has now been rewritten as

$$
\left|\begin{array}{cccc}
f_{1} & \ldots & f_{N} & \sum(-1)^{N+1+j} f_{j} \int p W\left(f_{1}, \ldots, \hat{f}_{j}, \ldots, f_{N+1}\right) \\
f_{1}^{\prime} & \ldots & f_{N}^{\prime} & \sum(-1)^{N+1+j} f_{j}^{\prime} \int p W\left(f_{1}, \ldots, \widehat{f}_{j}, \ldots, f_{N+1}\right) \\
\vdots & \vdots & & \vdots \\
f_{1}^{(N-1)} & \cdots & f_{N}^{(N-1)} & \sum(-1)^{N+1+j} f_{j}^{(N-1)} \int p W\left(f_{1}, \ldots, \hat{f}_{j}, \ldots, f_{N+1}\right) \\
f_{1}^{(N)} & \ldots & f_{N}^{(N)} & \sum(-1)^{N+1+j} f_{j}^{(N)} \int p W\left(f_{1}, \ldots, \hat{f}_{j}, \ldots, f_{N+1}\right)
\end{array}\right| .
$$

Fortunately, this is easy to evaluate. Almost all of the right-hand column can be removed from the determinant by subtracting multiples of the first $N$ columns. All that remains is

$$
\left|\begin{array}{cccc}
f_{1} & \cdots & f_{N} & f_{N+1} \int p W\left(f_{1}, \ldots, f_{N+1}\right) \\
f_{1}^{\prime} & \cdots & f_{N}^{\prime} & f_{N+1}^{\prime} \int p W\left(f_{1}, \ldots, f_{N+1}\right) \\
\vdots & \vdots & & \vdots \\
f_{1}^{(N-1)} & \cdots & f_{N}^{l N-1)} & f_{N+1}^{(N-1)} \int p W\left(f_{1}, \ldots, f_{N+1}\right) \\
f_{1}^{(N)} & \cdots & f_{N}^{(N)} & f_{N+1}^{(N)} \int p W\left(f_{1}, \ldots, f_{N+1}\right)
\end{array}\right|
$$

from which the proposition follows at once.

2.2 Using the Wronskian Relation. It is not immediately clear what, if anything, Corollary 1 has to do with finding recursion relations. However, let us notice several things. First, since (6) tells us that eigenfunctions after doing Darboux several times look like Wronskians, the left-hand side of (9) resembles $\Theta(x) \phi_{n}$, the multiplication of the eigenfunction by a function independent on $n$. This should give us the idea of what $\Theta$ will turn out to be.

The right-hand side of (9) is not so transparent. The way to think of it is that it is the result of applying an operator which is only dependent on the eigenvalue 
to the eigenfunction. Since the Wronskian for the eigenfunctions only has the eigenvalue appearing in the last term, this operator will pass through most of the Wronskian and not notice it. Its effect will only be on the last term. Thus, the right-hand side should correspond to $(B \phi)_{n}$.

It is important to emphasize that the identification suggested by the last paragraph certainly cannot always be done. There is no a priori reason to expect that the $B$ envisioned by such formal considerations will ever turn out to be a finite difference operator. Only in a few very special cases will we actually be able to see that this is so.

To work toward putting these reflections into practice: Let us say that $f_{1}, f_{2}, \ldots, f_{m}$ are eigenfunctions of an initial operator $L$, and that $\phi_{n}$ is an eigenfunction for the eigenvalue $\lambda_{n}$. If we have done Darboux $m$ times using eigenfunctions coming from $f_{1}, \ldots, f_{m},(6)$ tells us that a $\lambda_{n}$-eigenfunction of the final operator is of the form

$$
\psi_{n}=\frac{W\left(f_{1}, \ldots, f_{m}, \phi_{n}\right)}{W\left(f_{1}, \ldots, f_{m}\right)}
$$

As regards our Eq. (2),

$$
(B \psi)_{n}=\Theta(x) \psi_{n},
$$

it is clear that the denominator of (6), since it depends only on $x$, can be ignored completely - the operator $B$ will not notice it, nor will multiplication by $\Theta$. Henceforth, then, we will just think of the numerator

$$
\psi_{n} \sim W\left(f_{1}, f_{2}, \ldots, f_{m}, \phi_{n}\right)
$$

as representing the eigenfunction. Of course, if the $x$-equation (1) is being discussed, it will be essential to bring along the denominator as well.

In our Eq. (9), let $f_{m+1}$ be equal to $\phi_{n}$. Remember that only $f_{m+1}$ will depend at all on the eigenvalue $n$. We see at once that (9) is linear in $f_{m+1}=\phi_{n}$, and that the left-hand side is precisely $\Theta \psi_{n}$, where $\Theta \equiv \int p W\left(f_{1}, f_{2}, \ldots, f_{m}\right)$. What needs to be done is to represent the right-hand side as a difference operator acting on $\psi_{n}$.

Let us examine the last term of the right-hand side in detail:

$$
\sum(-1)^{j} f_{j} \int p W\left(f_{1}, \ldots, \hat{f}_{j}, \ldots, f_{m+1}\right),
$$

where $f_{m+1}=\phi_{n}$. This certainly doesn't look like a recursion operator in $n$ at this point! In fact, the operator acting here on $\phi_{n}=f_{m+1}$ depends only on $x$. It consists of some funny combination of multiplication by functions of $x$, differentiation, and integration with respect to $x$.

First we claim that all the differentiation of $f_{m+1}$ can be eliminated.

Lemma 5. Equation (10) can be rewritten without any derivatives of $f_{m+1}$.

Proof. All these derivatives appear inside integrals, and we will get rid of them by integration by parts. The only place for derivatives of $f_{m+1}$ to remain is in the boundary terms, and we will now show that all such vanish.

If all terms of $(10)$ that have a particular derivatives of $f_{m+1}$ inside the integral are collected, the result will typically be like 


$$
\pm \sum(-1)^{j} f_{j} \int p\left[\begin{array}{cccccc}
f_{1} & f_{2} & \cdots & \hat{f}_{j} & \cdots & f_{m} \\
0 & 1 & \cdots & \hat{\imath} & \cdots & m-1
\end{array}\right] f_{m+1}^{(i)} .
$$

Here the notation for the determinant from the beginning of the section is used. Now let us integrate by parts $i$ times to rid ourselves of all derivatives of $f_{m+1}$. Each boundary term will look like

$$
\pm p^{\left(i_{1}\right)} f_{m+1}^{\left(i_{3}\right)} \sum(-1)^{j} f_{j}\left[\begin{array}{cccccc}
f_{1} & f_{2} & \cdots & \hat{f}_{j} & \cdots & f_{m} \\
0 & 1 & \cdots & \hat{\imath} & \cdots & m-1
\end{array}\right]^{\left(i_{2}\right)}
$$

with $i_{1}, i_{2}, i_{3}<i$.

However, taking $i_{2}$ derivatives of determinants is something we know how to do. The result is a sum of determinants. In each term of the sum, $i_{2}$ rows, counting multiplicity, have been differentiated. This amounts here to adding a total of $i_{2}$ to the original $r_{k}$ 's, which were $0,1, \ldots, \hat{\imath}, \ldots, m-1$. In our case, since we multiply by $(-1)^{j} f_{j}^{(0)},(7)$ will force any such term to vanish unless 0 is not among the final $r_{k}$ 's. But in order that two of the $r_{i}$ 's should not be the same, at least $i$ derivatives are needed; for example, to move the original $r_{1}=0$ to $r_{1}=i$. Since we have only $i_{2}<i$ derivatives with which to work, this is impossible, and every term will vanish. This demonstrates the lemma.

It will be easier to see how to use all this to find the recursion relation from (9), given certain assumptions, if we do two real examples first. Therefore, the calculations will be done now in detail for $m=1$ and $m=2$ to demonstrate the procedures involved.

2.3. Darboux Once. The first case is where Darboux has been done precisely once, say with respect to some eigenfunction $f_{1}$ of the original $L$. The new eigenfunctions are $\psi_{n}=W\left(f_{1}, \phi_{n}\right) / f_{1}$. Here (9) says

$$
W\left(f_{1}, \phi_{n}\right) \int p f_{1}=W\left(f_{1},-f_{1} \int p \phi_{n}+\phi_{n} \int p f_{1}\right) .
$$

Define $\Theta \equiv \int p f_{1}$ and divide the equation by $f_{1}$, so that the left side becomes $\Theta \psi_{n}$.

Now say that the operation of multiplying by $x$ is some kind of difference (or pseudo-difference) operator in $n$. This means that there is some way of writing

$$
x \phi_{n}=X \phi_{n} \equiv \sum t_{m}^{n} \phi_{n+m},
$$

with all the $t_{m}^{n}$ 's independent of $x$. Here $X$ is a pseudo-difference operator, since there is no limit on the number of terms. Then our equation becomes

$$
\Theta \psi_{n}=\frac{p(X) W\left(f_{1},-f_{1} \int \phi_{n}\right)+\left(\int p f_{1}\right)(X) W\left(f_{1}, \phi_{n}\right)}{f_{1}} .
$$

Here the notation $g(X)$ means the pseudo-difference operator produced by the composition of $g$ with $X$.

If the operation of integration with respect to $x$ can also be represented as a pseudo-difference operator $D^{-1}$ in $n$-space, we can continue to unravel the equation into

$$
\Theta \psi_{n}=\frac{-p(X) D^{-1} W\left(f_{1}, f_{1} \phi_{n}\right)+\left(\int p f_{1}\right)(X) W\left(f_{1}, \phi_{n}\right)}{f_{1}}
$$


which in turn becomes

$$
\Theta \psi_{n}=\frac{p(X) D^{-1} f_{1}(X) W\left(f_{1}, \phi_{n}\right)+\left(\int p f_{1}\right)(X) W\left(f_{1}, \phi_{n}\right)}{f_{1}},
$$

and finally results in

$$
\left(\int p f_{1}\right)(x) \psi_{n}=\left(-(X) D^{-1} f_{1}(X)+\left(\int p f_{1}\right)(X)\right) \psi_{n} .
$$

Equation (11) gives us an explicit form for the expected $B$. The question remaining is, will this object be a finite recursion operator? The simplest situation where it will, and in truth the only case really known, is where the following conditions are satisfied:

1. $p$ is polynomial in $x$,

2. $f_{1}$ is also polynomial in $x$,

3. $X$ is a finite recursion operator, and

4. $D^{-1}$ is also a finite recursion operator, on the space of the $\phi_{n}$ 's.

In that case, it is quite certain that the operator

$$
B \equiv-p(X) D^{-1} f_{1}(X)+\left(\int p f_{1}\right)(X)
$$

will be a finite recursion operator. If $p$ and $f_{1}$ are polynomial of orders $a_{1}$ and $a_{2}$, and $X$ and $D^{-1}$ are finite recursion operators of orders $s_{1}$ and $s_{2}$ respectively, the operator $B$ can be seen to have order $\left(a_{1}+a_{2}\right) s_{1}+s_{2}$.

We can see from this calculation of the size of $B$ that it is not too easy to make very small $B$ 's. Reasonable starting places for both $X$ and $D^{-1}$ are both three-term (although $\mathrm{KdV}$ will be a notable exception). If we pick $p=1$ and $f_{1}$ to be linear - we could hardly make them smaller! - we still get a five-term recursion relation. Anything else will make things bigger and therefore perhaps less useful in practice.

It is important to discover the effect on (11) of changing the independent variable, or of multiplying the eigenfunctions by some $g(x)$ independent of $n$. These are morphisms 1 and 2 from Sect. 1.2. According to what we found there, $B$ should come out the same, yet all the parts of (11) seem to change. The key to understanding these transformations is in observing how they effect the operator $D^{-1}$.

First, consider morphism 2, and say we change the independent variable from $x$ to $y(x)$. An eigenfunction $\phi_{n}(x)$ will become a new one $\psi_{n}(x)=\phi_{n}(x(y))$. Then the new $D^{-1}$ will be given by

$$
\begin{aligned}
\widetilde{D^{-1}} \psi_{n}(y) & =\int \psi_{n}(y) d y=\int \phi_{n}(x) \frac{d y}{d x} d x \\
& =\frac{d y}{d x}(X) \int \phi_{n}(x) d x=\frac{d y}{d x}(X) D^{-1} \phi_{n}(x) .
\end{aligned}
$$

We can now see that setting the new $\tilde{p} \equiv p /(d y / d x)$ will make both terms of $B$ come out the same as before.

Now let us look at morphism 2, in which the new eigenfunctions are given by 


$$
\begin{aligned}
& \psi_{n}(x)=g(x) \phi_{n}(x) \text {. Then the new } \widetilde{D^{-1}} \text { is just } g(X) D^{-1} g^{-1}(X) \text { : } \\
& \qquad \begin{array}{r}
g(X) D^{-1} g^{-1}(X) \psi_{n}=g(x) g(X) D^{-1} g^{-1}(X) \phi_{n} \\
=g(X) D^{-1} \phi_{n}=g(X) \int \phi_{n}=\int g(x) \phi_{n}=\int \psi_{n}=D^{-1} \psi_{n} .
\end{array}
\end{aligned}
$$

Again, we can choose $\tilde{p} \equiv p / g$ to make the new $B$ the same as the old.

In the classical examples we have been considering, our notation does not always give the eigenfunctions in polynomial form. As we have seen, however, this does not matter; the two morphisms given here can be used to put the operator into a form with polynomial eigenfunctions, satisfying our four sufficient conditions. The $B$ found there will also be the right one for the original eigenfunctions.

Offhand, it might seem quite possible that a particular case could arise in which this Darboux procedure can be done, but only a few times. For example, perhaps our four conditions would be satisfied, but with only a few eigenfunctions being polynomials. However, a heuristic argument suggests that this is unlikely. If eigenfunctions $\phi_{i-1}$ and $\phi_{i}$ are polynomial, condition 4 requires that $\int \phi_{i}$, also a polynomial, must be a linear combination of $\phi_{i-1}, \phi_{i}$ and $\phi_{i+1}$. Thus, $\phi_{i+1}$ would be a polynomial also. Repeating this argument shows that there are then an infinite number of polynomial eigenfunctions, so that Darboux could be done arbitrarily many times. This is in fact the case in all the examples found here.

2.4. Multiple Darboux. This section should make clear how to do all higher-order examples in practice. By working out the example of doing two Darboux transformations we will see the use of the additional refinement from Lemma 5, necessary here, of getting rid of the derivatives.

Say that Darboux has been done twice, using $f_{1}$ and $f_{2}$. We get new eigenfunctions $\psi_{n} \equiv\left(W\left(f_{1}, f_{2}, \phi_{n}\right) / W\left(f_{1}, f_{2}\right)\right)$, and (9) takes the form

$$
\begin{aligned}
& W\left(f_{1}, f_{2}, \phi_{n}\right) \int p W\left(f_{1}, f_{2}\right) \\
& \quad=W\left(f_{1}, f_{2}, f_{1} \int p W\left(f_{2}, \phi_{n}\right)-f_{2} \int p W\left(f_{1}, \phi_{n}\right)+\phi_{n} \int p W\left(f_{1}, f_{2}\right)\right) .
\end{aligned}
$$

Let us examine the last term of the right-hand-side Wronskian in order to integrate it by parts and to unravel it:

$$
\begin{aligned}
f_{1} \int p W\left(f_{2}, \phi_{n}\right)-f_{2} \int p W\left(f_{1}, \phi_{n}\right)+\phi_{n} \int p W\left(f_{1}, f_{2}\right) \\
=f_{1} \int p\left(f_{2} \phi_{n}^{\prime}-f_{2}^{\prime} \phi_{n}\right)-f_{2} \int p\left(f_{1} \phi_{n}^{\prime}-f_{1}^{\prime} \phi_{n}\right)+\phi_{n} \int p W\left(f_{1}, f_{2}\right) \\
=-f_{1} \int\left(p^{\prime} f_{2}+2 p f_{2}^{\prime}\right) \phi_{n}+f_{2} \int\left(p^{\prime} f_{1}+2 p f_{1}^{\prime}\right) \phi_{n}+\phi_{n} \int p W\left(f_{1}, f_{2}\right) \\
=-\left(p^{\prime} f_{2}+2 p f_{2}^{\prime}\right)(X) D^{-1} f_{1}(X) \phi_{n}+\left(p^{\prime} f_{1}+2 p{f_{1}^{\prime}}_{1}\right)(X) D^{-1} f_{1}(X) \phi_{n} \\
\quad+\left(\int p W\left(f_{1}, f_{2}\right)\right)(X) \phi_{n} .
\end{aligned}
$$

This can be reinserted to the right-hand-side Wronskian of (12). If the equation is then divided by $W\left(f_{1}, f_{2}\right)$ and the difference operators pulled to the outside, the result is

$$
\begin{aligned}
\left(\int p W\left(f_{1}, f_{2}\right)\right)(x) \psi_{n}= & \left(-\left(p^{\prime} f_{2}+2 p f_{2}^{\prime}\right)(X) D^{-1} f_{1}(X)+\left(p^{\prime} f_{1}+2 p f_{1}^{\prime}\right)(X) D^{-1} f_{2}(X)\right. \\
& \left.+\left(\int p W\left(f_{1}, f_{2}\right)\right)(X)\right) \psi_{n},
\end{aligned}
$$

the required form for (2). 
Sufficient conditions for this $B$ to be a difference operator are similar to those for (11). If $X$ and $D^{-1}$ are both difference operators, and $p, f_{1}$, and $f_{2}$ are all polynomials, everything must work out. It is clear that such conditions will be the proper ones for Darboux done any number of times, and this will now be stated as a proposition:

Proposition 2. Assume that $L$ and its eigenfunctions $\phi_{n}$ satisfy (1) and (2). Assume also that integration of the eigenfunctions with respect to $x$ can be expressed also as a finite difference operator on the space of the $\phi_{n}$ 's. Then if the Darboux transformation is done $m$ times from $L$ using polynomial eigenfunctions $f_{1}, f_{2}, \ldots, f_{m}$, the new differential operator derived will also satisfy (1) and (2). Furthermore, for every polynomial $p(x)$ chosen, there will be $a B_{p}$ and $\Theta_{p}$ that can be used in (2). These are explicitly derived from (9).

If one insists on an $L$ of the particular form $\partial^{2}+V(x)$, very likely none of its eigenfunctions will actually be polynomials. However, this doesn't really matter, as we said above. It is enough that a change of variables with morphism 1 and conjugation with a function of $x$ by morphism 2 brings things into the conditions of this proposition.

For the sake of competeness, the $\Theta$ and $B$ for triple Darboux are included here. With the obvious notation, let

$$
\begin{aligned}
A_{1} \equiv & -2 p f_{2} f_{3}^{\prime \prime \prime}-3 p f_{2}^{\prime} f_{3}^{\prime \prime}-3 p^{\prime} f_{2} f_{3}^{\prime \prime}+3 p f_{2}^{\prime \prime} f_{3}^{\prime} \\
& -p^{\prime \prime} f_{2} f_{3}^{\prime}+2 p f_{2}^{\prime \prime \prime} f_{3}+3 p^{\prime} f_{2}^{\prime \prime} f_{3}+p^{\prime \prime} f_{2}^{\prime} f_{3}, \\
A_{2} \equiv & 2 p f_{1} f_{3}^{\prime \prime \prime}+3 p f_{1}^{\prime} f_{3}^{\prime \prime}+3 p^{\prime} f_{1} f_{3}^{\prime \prime}-3 p f_{1}^{\prime \prime} f_{3}^{\prime} \\
& +p^{\prime \prime} f_{1} f_{3}^{\prime}-2 p f_{1}^{\prime \prime \prime} f_{3}-3 p^{\prime} f_{1}^{\prime \prime} f_{3}-p^{\prime \prime} f_{1}^{\prime} f_{3}, \\
A_{3} \equiv & -2 p f_{1} f_{2}^{\prime \prime \prime}-3 p f_{1}^{\prime} f_{2}^{\prime \prime}-3 p^{\prime} f_{1} f_{2}^{\prime \prime}+3 p f_{1}^{\prime \prime} f_{2}^{\prime} \\
& -p^{\prime \prime} f_{1} f_{2}^{\prime}+2 p f_{1}^{\prime \prime \prime} f_{2}+3 p^{\prime} f_{1}^{\prime \prime} f_{2}+p^{\prime \prime} f_{1}^{\prime} f_{2},
\end{aligned}
$$

and

$$
A_{4} \equiv p W\left(f_{1}, f_{2}, f_{3}\right) \text {. }
$$

Then for the triple-Darboux case,

$$
\Theta=\left(\int A_{4}\right)(x)
$$

and

$$
B=A_{1}(X) D^{-1} f_{1}(X)+A_{2}(X) D^{-1} f_{2}(X)+A_{3}(X) D^{-1} f_{3}(X)+\left(\int A_{4}\right)(X) .
$$

The thorough reader who wishes to know the explicit form for quadruple-Darboux can jolly well figure it out for himself.

2.5. The Classical Examples. A complete classification of all functions satisfying three-term relations has already been published by the author [6], yielding only the classical functions. Can they be made to satisfy our requirements in order to be able to do this repeatable Darboux process? Happily, most of them can, in one way or another, giving us a large zoo of objects with recursion relations.

Start with eigenfunctions that already have three-term recursion relations. The conditions of Proposition 2 still needed are the polynomial eigenfunctions, with 
respect to which Darboux is done, and the finite difference relation for integration. To put the results into their simplest form, we will use the operators and eigenfunctions in their standard form, rather than the one we have been using. In the standard form, some of the eigenfunctions are actually polynomials, and the integral needs no extra Jacobian. These recursion relations for integration, where they exist, are included in Appendix A along with the usual recursion relations for these orthogonal polynomials. For some reason, these relations are not listed in usual tables, though they are generally not difficult to derive from listed formulas.

A final point of great importance: It is noted in Appendix A that for each of the classical examples, the difference operators $X$ and $D^{-1}$ actually have a full two-dimensional solution space. It is immediate that this property will be carried through all the manipulations of this chapter. The new eigenfunctions created by doing Darboux $m$ times are of the form

$$
\frac{W\left(f_{1}, f_{2}, \ldots, f_{m+1}\right)}{W\left(f_{1}, f_{2}, \ldots, f_{m}\right)} .
$$

If we are only careful to pick $f_{m+1}$ to fall in the full solution space of $X$ and $D^{-1}$, the derived $B$ for our new eigenfunctions will work perfectly. The only case known to have solution spaces that are only one-dimensional is the $\mathrm{N}$-soliton solution to $\mathrm{KdV}$, which will be considered in a separate article [8].

\section{Appendix A. The Classical Recursions}

Since it may be useful to have the recursion relations available in one place, in this section we give $X$ and, where it exists, $D^{-1}$, for solutions to our problem with three-term recursion relations. Many formulas will just be copied from Abramowitz and Stegun [1].

While the recursion relation for $X$ in most cases is generally well-known, the one for $D^{-1}$ is normally much harder to locate. It is perhaps worth noting the following: There is a well-known classical argument showing that, for orthogonal polynomials, the operator $X$ will always be a three-term difference operator. However, the same argument, using integration by parts, shows that $D^{-1}$ will also be a three-term operator for any set of orthogonal polynomials.

A.1. Hermite. The eigenfunctions here are solutions to the Hermite equation

$$
y^{\prime \prime}-2 x y^{\prime}+2 n y=0 .
$$

There is no problem in this case to find $X$ and $D^{-1}$. The former is given in [1], 22.7.13, by

and

$$
X=\frac{1}{2} S+n S^{-1}
$$

$$
D^{-1}=\frac{1}{2(n+1)} S
$$

is given in [1] as 22.13.16.

A.2. Laguerre. Use standard Generalized Laguerre polynomials, $\phi_{n} \equiv L_{n}^{(m)}(x)$. 
These solve

$$
x y^{\prime \prime}+(m+1-x) y^{\prime}+n y=0
$$

We have

$$
X=-(n+1) S+(2 n+m+1)-(n+m) S^{-1},
$$

from [1], 22.7.12. Since

$$
D X=n-(n+m) S^{-1},
$$

by $[1], 22.8 .6$, one recognizes easily that

so that

$$
X=(-S+1) D X
$$

$$
D^{-1}=-S+1
$$

which is [1], 22.13.14.

A.3. Bessel. The Bessel equation in its usual form,

$$
x^{2} y^{\prime \prime}+y^{\prime}+\left(x^{2}-n^{2}\right) y=0
$$

has a good three-term recursion relation for the eigenfunctions. We need also, though, to find eigenfunctions that are polynomial. This is not quite so simple, since the Bessel functions that everyone uses are not polynomial at all. However, polynomials can be found; these come from the Hankel functions at half-integral eigenvalues, which are also solutions to the Bessel equation. From [1], 10.1.1, 10.1.16, and 10.1.17, we see that

$$
\begin{aligned}
H_{n+1}^{(1)}(z) & =\sqrt{\frac{2}{\pi z}} i^{(n+1)} e^{i z} \sum_{k=0}^{n}(n+1 / 2, k)(-2 i z)^{-k}, \\
H_{n+1 / 2}^{(2)}(z) & =\sqrt{\frac{2}{\pi z}} i^{-(n+1)} e^{-i z} \sum_{k=0}^{n}(n+1 / 2, k)(2 i z)^{-k} .
\end{aligned}
$$

Here

$$
(n+1 / 2, k) \equiv(n+k) ! /(k !(n-k) !)
$$

We can get polynomials by letting $x=1 / z$, and dropping all the $n$-independent stuff in front of the sums, giving

$$
\phi_{n}^{ \pm} \equiv i^{ \pm n} \sum_{k=0}^{n}(n+1 / 2, k)(\mp 2 i)^{-k} x^{k}
$$

Since the Bessel functions all satisfy [1], 9.1.27,

$$
\frac{1}{2 n} C_{n+1}(z)+\frac{1}{2 n} C_{n-1}(z)=\frac{1}{z} C_{n}(z)
$$

these new eigenfunctions will satisfy

$$
\frac{1}{2 n+1} \phi_{n+1}+\frac{1}{2 n+1} \phi_{n-1}=x \phi_{n},
$$


as we've shifted $n$ by $1 / 2$. Thus

$$
X=\frac{1}{2 n+1} S+\frac{1}{2 n+1} S^{-1}
$$

Now according to $[1], 9.1 .27$,

$$
\frac{d}{d z} H_{n}=\frac{1}{2} H_{n-1}-\frac{1}{2} H_{n+1}
$$

so

$$
\begin{aligned}
& \left(\frac{1}{2 \sqrt{z}} e^{ \pm i z} \phi_{n-1}^{ \pm}-\frac{1}{2 \sqrt{z}} e^{ \pm i z \phi_{n+1}^{ \pm}}\right) \\
& \quad=\frac{d}{d z}\left(\frac{1}{\sqrt{z}} e^{ \pm \imath z} \phi_{n}^{ \pm}\right)=\left(\frac{-1}{2 z} \pm i\right)\left(\frac{1}{\sqrt{z}} e^{ \pm i z} \phi_{n}^{ \pm}\right)+\frac{1}{\sqrt{z}} e^{ \pm \imath z} \frac{d}{d z} \phi_{n}^{ \pm} .
\end{aligned}
$$

Now let $x=1 / z$, so that $d / d z=-x^{2}(d / d x)$. The equation simplifies to

$$
\frac{1}{2} \phi_{n-1}^{ \pm}-\frac{1}{2} \phi_{n+1}^{ \pm}=\left(-\frac{x}{2} \pm i\right) \phi_{n}^{ \pm}-x^{2} \frac{d}{d x} \phi_{n}^{ \pm} .
$$

One sees that here

$$
D X^{2}=\frac{1}{2} S-\frac{1}{2} X \pm i-\frac{1}{2} S^{-1}=\frac{n}{2 n+1} S \pm i-\frac{n+1}{2 n+1} S^{-1} .
$$

Now, multiplication on the left by $D^{-1}$ yields

$$
X^{2}=D^{-1}\left(\frac{n}{2 n+1} S \pm i-\frac{n+1}{2 n+1} S^{-1}\right) .
$$

Squaring our equation for $X$ gives

$$
X^{2}=\frac{1}{(2 n+1)(2 n+3)} S^{2}+\frac{2}{(2 n-1)(2 n+3)}+\frac{1}{(2 n-1)(2 n+1)} S^{-2} .
$$

Factoring the last equation gives us our desired

$$
D^{-1}=\frac{1}{(n+1)(2 n+1)} S \mp \frac{i}{n(n+1)}-\frac{1}{n(2 n+1)} S^{-1},
$$

where the $\mp$ depends on whether we're using $\phi_{n}^{+}$or $\phi_{n}^{-}$.

A.4. Double Bessel. The eigenfunctions for the Bessel equation also satisfy a different three-term recursion relation of the proper form. Since the previous $X$ had no $S^{0}$ term, its square in (14) is missing the terms for $S$ and $S^{-1}$. Therefore, redefining the eigenvalue parameter as $n^{\prime} \equiv 2 n$, we get a new difference operator, of the following form:

$$
\frac{1}{(2 n+1)(2 n+3)} S+\frac{2}{(2 n-1)(2 n+3)}+\frac{1}{(2 n-1)(2 n+1)} S^{-1},
$$


here with the old $S^{2}$ replaced by $S$. In this example, we would work with $x=1 / z^{2}$ in the Hankel functions. However, this is the first case where there seems to be nowwhere to go, for there are no polynomial eigenfunctions associated with this variable. The ones used for the previous example were not functions of $1 / z^{2}$, but of $1 / z$, but of $1 / z$, and so they will have $\sqrt{x}$ terms here. Thus, we cannot make any Darboux steps from this starting point.

A.5. Special Laguerre. Another three-term relation exists for the Generalized Laguerre equation. Though this case works out very nicely, it is not so easy to calculate the required recursion relations by hand. The author found it much simpler to derive them from the formal series definition of the functions than by manipulating the known relationships of the Laguerre functions. Therefore, only results will be given, which can be readily checked after the fact against the formal series.

The necessary polynomial eigenfunctions are found to be in the form

$$
\phi_{n}^{m}(x)=x^{n} L_{n}^{(m-2 n)}\left(\frac{1}{x}\right)
$$

Thus, they are not solutions to the Laguerre equation itself, but of the result of several of the morphisms from Sec. 1.2. These functions are obtained from normal Laguerre functions by letting $x$ become $1 / x, n$ become $-n-(m+1) / 2$, and by dropping an $n$-independent function from in front of the eigenfunctions. Our $\phi_{n}$ 's are certainly polynomials for $n$ any non-negative integer, since $L_{n}^{\alpha}(1 / x)$ is only an $n^{\text {th }}$-degree polynomial in $1 / x$. The formal expression for the $\phi_{n}$ 's is

$$
\phi_{n}^{m}(x) \equiv x^{n} L_{n}^{(m-2 n)}\left(\frac{1}{x}\right)=\sum_{k=0}^{n}(-1)^{k}\left(\begin{array}{c}
n+m \\
n-k
\end{array}\right) \frac{1}{k !} x^{n-k}
$$

from [1], 22.3.9. It is not too hard to check now that

$$
\begin{aligned}
X= & -\frac{(n+1)(n-m)}{(2 n-m)(2 n-m+1)} S \\
& +\frac{m+1}{(2 n-m-1)(2 n-m+1)}+\frac{1}{(2 n-m-1)(2 n-m)} S^{-1}
\end{aligned}
$$

and that

$$
\begin{aligned}
D^{-1}= & -\frac{n-m}{(2 n-m)(2 n-m+1)} S \\
& +\frac{2}{(2 n-m-1)(2 n-m+1)}-\frac{1}{(n-m-1)(2 n-m-1)(2 n-m)} S^{-1} .
\end{aligned}
$$

A.6. Jacobi. This is the largest class of functions with three-term relations. Its working out nicely provides very many ways to do Darboux steps. The polynomials to be used are $P_{n}^{(\alpha, \beta)}(x)$, defined in [1], 22.3.1. They are solutions to

$$
\left(1-x^{2}\right)\left(P_{n}^{\alpha, b e}\right)^{\prime \prime}+(\beta-\alpha-(\alpha+\beta+2) x)\left(P_{n}^{\alpha, \beta}\right)^{\prime}+n(\alpha+\beta+n+1) P_{n}^{\alpha, \beta}=0 .
$$


By [1], 22.7.1,

$$
\begin{aligned}
X= & \frac{2(n+1)(n+\alpha+\beta+1)}{(2 n+\alpha+\beta+1)(2 n+\alpha+\beta+2)} S \\
& -\frac{\alpha^{2}-\beta^{2}}{(2 n+\alpha+\beta)(2 n+\alpha+\beta+2)}+\frac{2(n+\alpha)(n+\beta)}{(2 n+\alpha+\beta)(2 n+\alpha+\beta+1)} S^{-1} .
\end{aligned}
$$

Now

$$
\int P_{n}^{(\alpha, \beta)} d x=\frac{2}{n+\alpha+\beta} P_{n+1}^{(\alpha-1, \beta-1)}
$$

from its explicit formula [1], 22.3.1. Use [1], 22.7.18,

$$
P_{n}^{(\alpha-1, \beta)}=\frac{n+\alpha+\beta}{2 n+\alpha+\beta} P_{n}^{(\alpha, \beta)}-\frac{n+\beta}{2 n+\alpha+\beta} P_{n-1}^{(\alpha, \beta)}
$$

and [1], 22.7.19,

$$
P_{n}^{(\alpha, \beta-1)}=\frac{n+\alpha+\beta}{2 n+\alpha+\beta} P_{n}^{(\alpha, \beta)}+\frac{n+\beta}{2 n+\alpha+\beta} P_{n-1}^{(\alpha, \beta)},
$$

to put the $(\alpha, \beta)$ back to where they started from. The result is

$$
\begin{aligned}
D^{-1}= & \frac{2(n+\alpha+\beta+1)}{(2 n+\alpha+\beta+1)(2 n+\alpha+\beta+2)} S+\frac{2(\alpha-\beta)}{(2 n+\alpha+\beta)(2 n+\alpha+\beta+2)} \\
& -\frac{2(n+\alpha)(n+\beta)}{(n+\alpha+\beta)(2 n+\alpha+\beta)(2 n+\alpha+\beta+1)} S^{-1} .
\end{aligned}
$$

It is clear that, although forms such as this one are pretty, it will be difficult to get explicit recursion relations for this case, having done Darboux seveal times.

A.7. Note on Solution Spaces. The following fact will be stated without proof: In all of these examples, including both of the families of polynomials for Bessel, the relevant difference equations have a full two-dimension solution space. That is, if a given difference operator $X$ or $D^{-1}$ is the correct one for the eigenfunctions $\phi_{n}$, we can also find independent eigenfunctions $\psi_{n}$ such that $(X \psi)_{n}=x \psi_{n}$ and $\left(D^{-1} \psi\right)_{n}=\int \psi_{n}$. Since the usual books of tables don't give many properties of the full solution spaces, this has to be checked by hand. The easiest way to derive it is just to find the power series expansion for an independent eigenfunction $\psi_{n}$, and see that it can be made to satisfy the same difference equations. In each case, it is likely to be necessary to multiply $\psi_{n}$ by the proper function of $n$ alone, giving $t_{n} \psi_{n}(x)$, say. Otherwise, instead of having difference equations involving the same $X$ and $D^{-1}$ as for the other solution, its difference operators will likely be these, but conjugated by morphism 3 .

\section{References}

1. Handbook of mathematical functions. Abramowitz M., Stegun, I.,1 (eds.). Washington, DC: National Bureau of Standards, US Government Printing Office, 1970 
2. Adler, M., Moser, J.: On a class of polynomials connected with the Korteweg-de Vries equations. Commun. Math. Phys. 61, 1-30 (1978)

3. Burchnall, J. L., Chaundy, T. W.: Commutative ordinary differential operators. Proc. Lond. Math. Soc. 21, 420-440 (1923)

4. Darboux, G.: Lecons sur la theorie generale des surfaces. 2ème partie. Paris: Gauthiers-Villars 1889

5. Duistermaat, J. J., Grünbaum, F. A.: Differential equations in the spectral parameter. Commun. Math. Phys. 103, 177-240 (1986)

6. Reach, M.: Recursion relations for solutions to the Schödinger Equation SIAM J. Math. Anal. 18, 465-472 (1987)

7. Reach, M.: Classification of recursion relations in the eigenvalue, preprint

8. Reach, M.: Difference equations for $N$-soliton solutions to $\mathrm{KdV}$, preprint

Communicated by J. N. Mather

Received October 23, 1987 